\title{
Erratum for: "Effect of JJYMD-C, a novel synthetic derivative of gallic acid, on proliferation and phenotype maintenance in rabbit articular chondrocytes in vitro" [Braz J Med Biol Res (2014) 47(8): e3935]
}

\section{Erratum for: Braz J Med Biol Res | doi: 10.1590/1414-431X20143935}

The Authors would like to correct Figure 6 and Figure 8 that were published incorrectly in the article "Effect of JJYMD-C, a novel synthetic derivative of gallic acid, on proliferation and phenotype maintenance in rabbit articular chondrocytes in vitro" in volume 47 no. 8 (2014) of the Brazilian Journal of Medical and Biological Research <http://dx.doi.org/10.1590/ $1414-431 \times 20143935>$.

The images of cell viability of $0.125 \mu \mathrm{g} / \mathrm{mL}$ JJYMD-C at 2 days in Figure 6 and of immunohistochemical staining for type II collagen of control at 2 days in Figure 8 were mistakenly submitted. The correct Figures 6 and 8 are published below. The authors apologize to the readers and to the Brazilian Journal of Medical and Biological Research.

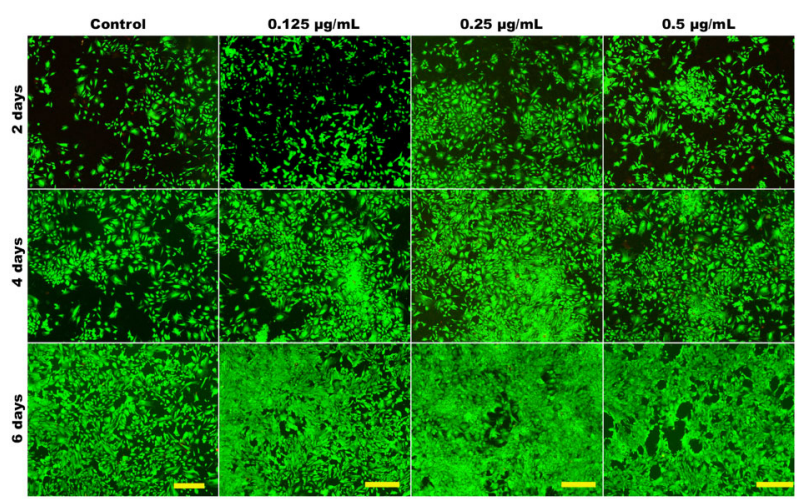

Figure 6. Laser-scanning confocal microscopy images showing the viability of chondrocytes cultured in vitro with 0 (control), $0.125,0.25$, and $0.5 \mathrm{mg} / \mathrm{mL}$ JJYMD-C for 2,4 , and 6 days. Scale bar: $100 \mathrm{~mm}$.

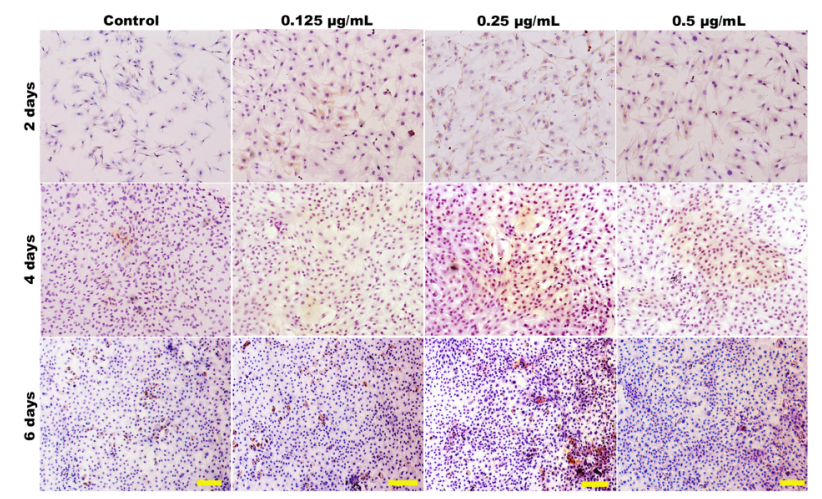

Figure 8. Immunohistochemical staining revealed the presence of type II collagen. Chondrocytes cultured in vitro with 0 (control), $0.125,0.25$, and $0.5 \mathrm{mg} / \mathrm{mL}$ JJYMD-C for 2,4 , and 6 days. Scale bar: $100 \mathrm{~mm}$. 